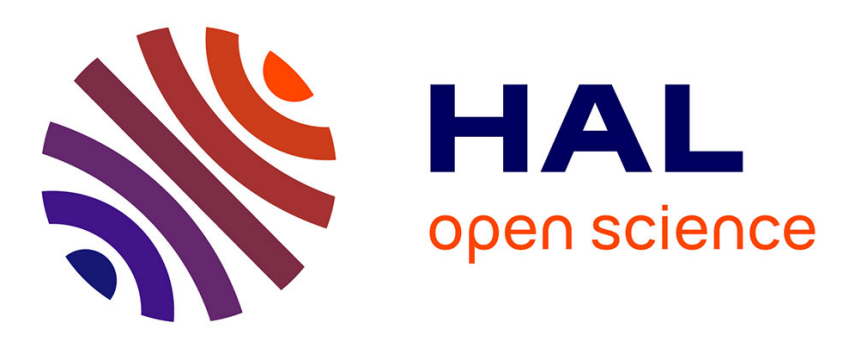

\title{
Motion Control of Cable-Driven Continuum Catheter Robot through Contacts
}

Zhongkai Zhang, Jeremie Dequidt, Junghwan Back, Hongbin Liu, Christian Duriez

\section{- To cite this version:}

Zhongkai Zhang, Jeremie Dequidt, Junghwan Back, Hongbin Liu, Christian Duriez. Motion Control of Cable-Driven Continuum Catheter Robot through Contacts. IEEE Robotics and Automation Letters, 2019, 4 (2), pp.1852-1859. 10.1109/LRA.2019.2898047 . hal-02052637

\section{HAL Id: hal-02052637 https://hal.science/hal-02052637}

Submitted on 28 Feb 2019

HAL is a multi-disciplinary open access archive for the deposit and dissemination of scientific research documents, whether they are published or not. The documents may come from teaching and research institutions in France or abroad, or from public or private research centers.
L'archive ouverte pluridisciplinaire $\mathbf{H A L}$, est destinée au dépôt et à la diffusion de documents scientifiques de niveau recherche, publiés ou non, émanant des établissements d'enseignement et de recherche français ou étrangers, des laboratoires publics ou privés. 


\title{
Motion Control of Cable-Driven Continuum Catheter Robot through Contacts
}

\author{
Zhongkai Zhang ${ }^{1}$, Jeremie Dequidt ${ }^{1}$, Junghwan Back ${ }^{2}$, Hongbin Liu $^{2}$, Christian Duriez ${ }^{1}$
}

\begin{abstract}
Catheter-based intervention plays an important role in minimally invasive surgery. For the closed-loop control of catheter robot through contacts, the loss of contact sensing along the entire catheter might result in task failure. To deal with this problem, we propose a decoupled motion control strategy which allows to control insertion and bending independently. We model the catheter robot and the contacts using the Finite Element Method. Then, we combine the simulated system and the real system for the closed-loop motion control. The control inputs are computed by solving a quadratic programming (QP) problem with a linear complementarity problem (LCP). A simplified method is proposed to solve this optimization problem by converting it into a standard QP problem. Using the proposed strategy, not only the control inputs but also the contact forces along the entire catheter can be computed without using force sensors. Finally, we validate the proposed methods using both simulation and experiments on a cabledriven continuum catheter robot for the real-time motion control through contacts.
\end{abstract}

\section{INTRODUCTION}

Robotic catheter systems eliminate radiation exposure to surgeons by allowing to be operated remotely. In the past decade, both manual and automatic steerable systems have been designed to increase the safety and the procedural effectiveness. However, the precise steering using a more accurate, safer, and more reliable approach still remains to be one of the main challenges for catheter insertion.

Unlike rigid robots, catheter robots are soft with infinite degrees of freedom so that the accurate modeling of catheter robots is difficult, especially in a constrained environment. In the literature, several methods have been proposed to model catheter robots. One approach employs the pseudorigid-body (PRB) model which consists of a set of rigid links connected with rotation joints [1], [2]. In order to capture more accurate deformation, Cosserat rod theory is used to model the 3-D static deflection of catheters [3], [4]. Another method is proposed to model the catheter with the assumption of constant curvature [5]. Catheters can also be modeled as multiple beams and simulated using Finite Element Method (FEM) [6]. FEM is an effective strategy to simulate the 3D deformation of structures with external forces. Using the realtime FEM technology, computational cost is dramatically

This work was supported by the Region Hauts-de-France, the project COMOROS (ERDF funds), and EPSRC UK-grant No: EP/R013977/1.

1. Z. Zhang, J. Dequidt and C. Duriez are affiliated with INRIA and University of Lille, France. Contact: \{zhongkai.zhang, jeremie.dequidt, christian.duriez\}@inria.fr.

2. J. Back and H. Liu are affiliated with King's College London, UK. Contact: \{junghwan.back, hongbin.liu\}@kcl.ac.uk. reduced (The model can be updated at 30 to $50 \mathrm{~Hz}$.) for the interactive simulation.

Catheter robots can be controlled using a model based on the assumption of constant curvature [7]. Most position controllers are designed based on the inverse kinematic model so that the computation of inverse Jacobian matrix is indispensable. Jacobian matrix can be computed based on either the analytical model [7], [8] or the numerical model [9], [10]. These methods are proposed for the position control of catheters or soft robots without considering the interaction with the environment. The loss of contact information might result in imprecise steering and even the failure of the task because of the unsensed Jacobian matrix rotation [11]. The position of catheter tip can also be controlled by the modelless [12] (Jacobian matrix is estimated empirically using the input and output data) or the model-free [13] control strategies. Although they are robust to the environment, contact forces along the entire robot are difficult to be captured.

The available force feedback during catheter insertion is an important strategy to avoid damaging the blood vessel by applying excessive force. The contact forces that the catheter tip applies on the blood vessel can be measured by an embedded force sensor [14], [15] or by an indirect force measurements [16], [17], [18] (like the image-based force sensing). However, none of the available strategies is capable of providing the catheter-tissue interaction forces along the entire catheter. There is, therefore, a need to report sufficient information of contact forces to the surgeon by visual or haptic feedback to increase the safety of catheter insertion. Given the known environment and catheter model, the location of contact forces is detected by collision detection algorithm and the intensity along the entire catheter can be computed by the FEM [6]. This strategy is widely used for medical simulation where the contact is modeled based on the Signorini's law [19], [20].

To achieve the closed-loop control, the catheter robot and the contacts are simulated in a real-time simulator and are combined with the real system. The simulated system provides the Jacobian matrix and detects the collision between the robot and its environment. The contact is usually modeled as a linear complementarity problem (LCP). Then, the motion control with contacts can be converted as a quadratic programming (QP) problem with a LCP. Treating the contact forces as optimization variables is employed for both the rigid robot [21] and the soft manipulator [22]. The computation of the QP problem with LCP is difficult and it is necessary to 
explore a simpler strategy to solve this optimization problem.

This work aims to provide a possible strategy to increase the safety of catheter robots by independent control of insertion and bending as well as visualization of contracts forces along the catheter. Three main contributions are shown in this paper: (1) A closed-loop decoupled control strategy is proposed for catheter robot. It allows the surgeon to remain in direct control of the catheter insertion; (2) The contact forces are computed along the entire robot by solving a $\mathrm{QP}$ problem with LCP; (3) We proposed a simplified strategy to solve the problem of QP with LCP where the LCP holds strictly for a reachable trajectory.

This paper is organized as follows. Section II introduces the approach of quasi-static modeling of catheter robots with contacts based on real-time FEM. The problem statement and the overview of the proposed strategy is shown in Section III. The closed-loop decoupled control is proposed in Section IV where the bending and insertion are decoupled. The simulation-based validation and experiments are presented in Section V. Finally, conclusion and future work are shown in Section VI.

\section{QUASI-STATIC MODELING OF CATHETER ROBOTS WITH CONTACTS BASED ON REAL-TIME FEM}

The quasi-static equilibrium equation of catheter robots is deduced based on the real-time FEM without considering the dynamic feature (like the inertia force).

$$
\mathbf{f}_{\text {ext }}+\mathbb{F}(\mathbf{x})+\mathbf{H}_{a}^{T} \lambda_{a}+\mathbf{H}_{c}^{T} \lambda_{c}=0
$$

where $\mathbf{x} \in \mathbb{R}^{n}$ ( $n$ is the total degrees of freedom of all nodes) is the position vector of all nodes and is updated according to the configuration of the catheter robots at each sampling step. $\lambda_{a} \in \mathbb{R}^{a}$ ( $a$ is the number of actuators) and $\lambda_{c} \in \mathbb{R}^{c}$ ( $c$ is the number of contact points) are the constraint force of actuators and contacts, respectively. The directions of the forces on the nodes exerted by actuators and contacts are provided by $\mathbf{H}_{a}^{T} \in \mathbb{R}^{n \times a}$ and $\mathbf{H}_{c}^{T} \in \mathbb{R}^{n \times c}$. Therefore, $\mathbf{H}_{a}^{T} \lambda_{a}$ and $\mathbf{H}_{c}^{T} \lambda_{c}$ represent respectively the contributions of the actuators and the contacts to the position of FEM nodes. $\mathbf{f}_{\text {ext }} \in \mathbb{R}^{n}$ represents the external loads (like the gravity) and $\mathbb{F}(\mathbf{x}) \in \mathbb{R}^{n}$ gathers the internal stiffness forces.

The internal stiffness forces $\mathbb{F}(\mathbf{x})$ can be expressed by its first-order Taylor expansion:

$$
\mathbb{F}(\mathbf{x}+d \mathbf{x}) \approx \mathbb{F}(\mathbf{x})+\mathbf{K}(\mathbf{x}) d \mathbf{x}
$$

where $\mathbf{K}(\mathbf{x}) \in \mathbb{R}^{n \times n}$ is the tangent stiffness matrix which is highly sparse and depends on the actual position of the nodes $\mathbf{x} . d \mathbf{x}$ is the displacement vector of FEM nodes between consecutive configurations.

Substituting (2) into (1), the linearization of (1) per simulation step can be obtained:

$$
\mathbf{K}(\mathbf{x}) d \mathbf{x}=\mathbf{f}_{e x t}+\mathbb{F}(\mathbf{x}+d \mathbf{x})+\mathbf{H}_{a}^{T} \lambda_{a}+\mathbf{H}_{c}^{T} \lambda_{c}
$$

We define $\delta_{e} \in \mathbb{R}^{3 e}$ ( $e$ is the number of effectors), $\delta_{a} \in \mathbb{R}^{a}$ and $\delta_{c} \in \mathbb{R}^{c}$ as respectively the positions of effectors (the tip of the catheter robot), the displacement of actuators (the change of cables' length), and the interpenetration gap of contacts.
Based on the Signorini's law, for every contact point, there is a complementarity relation between the interpenetration gap $\delta_{c}$ and the contact force $\lambda_{c}$ among the normal direction. The complementarity relation can be written as [19]:

$$
\mathbf{0} \leq \delta_{c} \perp \lambda_{c} \geq \mathbf{0}
$$

The complementarity condition (4) can also be formulated as $\delta_{c} \geq 0, \lambda_{c} \geq 0$ and $\delta_{c}^{T} \lambda_{c}=0$. The contact positions can be detected using a collision detection algorithm. Then, the contact forces are computed by solving (4).

The computation of (3) consists of two steps [23]: (1) the computation of a free configuration $\mathbf{x}^{\text {free }}$ considering that there is no actuation and no contact; (2) the computation of $\lambda_{a}$ and $\lambda_{c}$ by solving an optimization problem.

Based on (3), the optimization in the motion space is timeconsuming because the size of matrix $\mathbf{K}$ is usually very large. By projecting the equilibrium equation (3) into the constraint space $^{1}$, the dimension of the matrices can be dramatically reduced so that the control inputs can be computed in realtime [23]. The projected equations can be written as:

$$
\begin{aligned}
& \delta_{e}=\mathbf{W}_{e} \lambda+\delta_{e}^{\text {free }} \\
& \delta_{a}=\mathbf{W}_{a} \lambda+\delta_{a}^{\text {free }} \\
& \delta_{c}=\mathbf{W}_{c} \lambda+\delta_{c}^{\text {free }}
\end{aligned}
$$

where $\mathbf{W}_{i}=\left[\begin{array}{ll}\mathbf{W}_{i a}(\mathbf{x}) & \mathbf{W}_{i c}(\mathbf{x})\end{array}\right]$ with $i$ standing for the subscripts $e$ (effector), $a$ (actuator) and $c$ (contact), respectively. $\mathbf{W}_{e} \in \mathbb{R}^{e \times(a+c)}, \mathbf{W}_{a} \in \mathbb{R}^{a \times(a+c)}$ and $\mathbf{W}_{c} \in \mathbb{R}^{c \times(a+c)}$ are compliance matrices which show the mechanical coupling between the effectors, actuators and contacts. The method to compute the compliance matrices is introduced in [23]. The external forces applied on the FEM can be written as $\lambda=\left[\begin{array}{ll}\lambda_{a} & \lambda_{c}\end{array}\right]^{T} \in \mathbb{R}^{a+c}$. The free motion vectors $\delta_{e}^{\text {free }} \in \mathbb{R}^{3 e}, \delta_{a}^{\text {free }} \in \mathbb{R}^{a}$ and $\delta_{c}^{\text {free }} \in \mathbb{R}^{c}$ are computed by $\delta_{i}^{\text {free }}=\delta_{i}(x)+H_{i} d x^{\text {free }}(i=a, b, c)$ with the assumption of $\lambda=0$.

At the next step, the configurations of both the catheter robot and the contacts are updated by

$$
d \mathbf{x}=\mathbf{K}^{-1}\left[\begin{array}{ll}
\mathbf{H}_{a}^{T} & \mathbf{H}_{c}^{T}
\end{array}\right] \cdot \lambda+\mathbf{d x}^{\text {free }}
$$

Remark 1. Compliance matrices $\mathbf{W}_{e}$ and $\mathbf{W}_{c}$ depend on the configuration of catheter robot so that they change at each iteration and can not be pre-computed. If the actuators and contacts act on different FEM nodes or with different directions, the matrices $\mathbf{W}_{e}$ has a full row rank $(e \leq(a+c))$ or a full column rank $(e \geq(a+c))$, and $\mathbf{W}_{c}$ has a full row rank.

\section{PROBLEM STATEMENT AND OVERVIEW OF PROPOSED CONTROL STRATEGY}

In this section, we first introduce the problems for the control design without considering the contacts. Then, we

\footnotetext{
${ }^{1}$ The constraint space is defined by the effectors, actuators and contacts (they are treated as constraint for the FE model). The details are shown in our former work [23].
} 
give an overview of the decoupled motion control strategy for a cable-actuated catheter robot through contacts.

\section{A. Problems for control design without considering contact}

As shown in Fig. 1 which is first introduced in [11], there are two canonical problems (artificial singularity and inverted mapping) for the cable-driven continuum robot if contacts exist on the robot but are not considered for the closed-loop control design. In the worst case, unsensed contacts would result in a positive-feedback loop. Both the artificial singularity and the inverted mapping are generated by the coupling between the insertion and bending. Therefore, to avoid the two canonical problems (shown in Fig. 1), we propose a decoupled control strategy by decoupling the insertion and the bending. Besides, the decoupled implementation of insertion and bending provides a possible solution to increase the safety for catheter insertion and to allows the surgeon to remain in direct control of the catheter insertion.

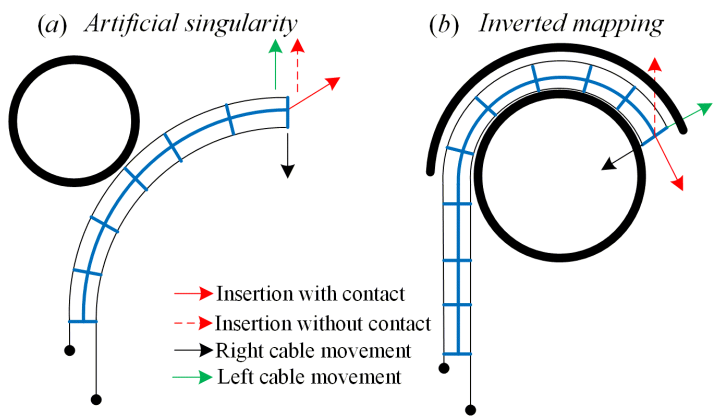

Fig. 1. Two canonical problems for the closed-loop control design [11]. (a) artificial singularity and $(b)$ inverted mapping result in the deterioration and even the positive-feedback loop.

\section{B. Decoupled closed-loop bending control}

The proposed closed-loop strategy (see Fig. 2) needs to combine both the catheter robot and its FE model which is simulated in real-time. The FE model is employed to compute the Jacobian matrix and to detect collisions between the robot and the environment. The same insertion input $\delta_{\text {in }}$ is sent to both the catheter robot and its FE model.

The bending control strategy is implemented based on the equations in $2 \mathrm{D}$ space converted by the projection module. Both the catheter robot and its FE model are controlled to follow the same predefined trajectories $\left(\delta_{d}\right.$ and $\delta_{d}^{F E M}$ respectively). For the actuation of FE model, the control inputs and the contact forces are computed by solving a quadratic programming $(\mathrm{QP})$ problem with a linear complementarity constraint. Using the decoupled strategy, the robot controller is designed without taking contacts into consideration, which aims to simplify the control design and to avoid the impacts of imprecise contact model.

\section{DECOUPLED CONTROL}

In this section, we introduce the design of the three components (Projection, FEM Controller and Robot Controller) needed for the decoupled control of catheter robots.

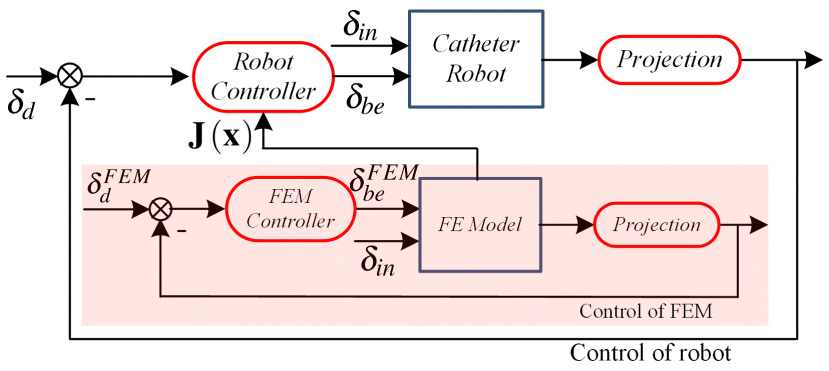

Fig. 2. Framework of decoupled closed-loop bending control for catheter robot through contacts. For the implementation, the FE model of catheter robot and its environment is simulated in real-time. The insertion and bending are decoupled by the projection block which is introduced in Section IV.A. Two independent controllers are employed for the control of catheter robot and its FE model respectively. $\delta_{d}$ and $\delta_{b e}$ are respectively the desired position and the bending control inputs for the catheter robot, while $\delta_{d}^{F E M}$ and $\delta_{b e}^{F E M}$ are the corresponding variables for the FE model. $\delta_{i n}$ is the insertion input for both the catheter robot and the FE model.

\section{A. Projection design}

The projection component is used to decouple the insertion and the bending for the catheter robot. As shown in Fig. 3, the movement of catheter robot is modeled in 3D space defined by the coordinate system $O X Y Z$. To achieve the projection, the local coordinate system Pxyz is computed at each step based on the 3D tip position and the predefined trajectory so that it moves with the insertion of catheter robot. After the projection process, the movement of catheter tip in 3D space is converted to the insertion along the tangent axis $x$ and the bending movement in $2 \mathrm{D}$ plane defined by the normal axis $z$ and the projection axis $y$.

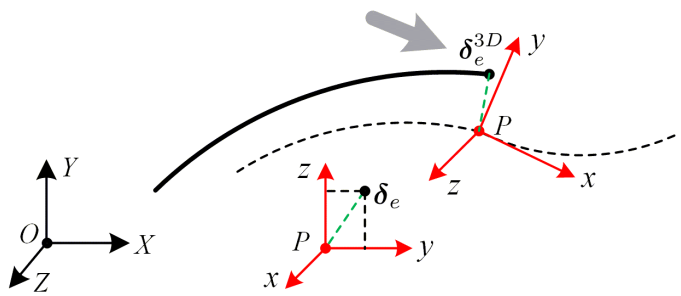

Fig. 3. Projection strategy for the catheter robot. The 3D position of the catheter tip in the global coordinate system $O X Y Z$ is projected to be the $2 \mathrm{D}$ position in the local coordinate system Pxyz. $P$ is the nearest point on the desired trajectory to the catheter tip $\delta_{e}^{3 D} . P x$ is the tangent vector for the trajectory at the point $P . P y$ and $P z$ are respectively the projection vector and the normal vector.

We project the 3D equations (5), (6) and (7) on a moving 2D plane orthogonal to the tip direction. This projection provides equations for the control of the bending movement ${ }^{2}$. The algorithm for the projection component design is summarized in Algorithm 1. During the bending control design, the desired position of catheter tip is the nearest point $P$ on the predefined trajectory. Then the 3D position error $\delta_{e}^{3 D} P$ is projected to a $2 \mathrm{D}$ vector in the plan $P y z$ and its norm is minimized with the bending controller.

\footnotetext{
${ }^{2}$ To simplify the notation, the equations (5), (6) and (7) in the following of this paper are used to capture the motion in $2 \mathrm{D}$ space.
} 


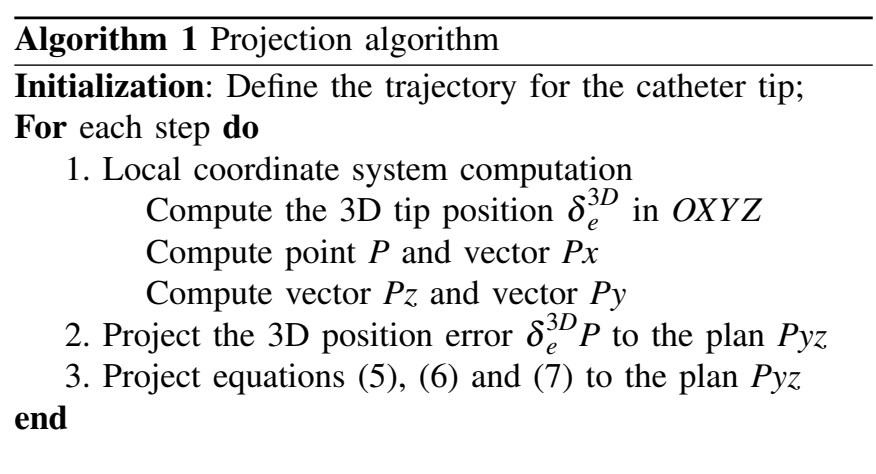

For the implementation of the bending control, both the FEM controller and the Robot controller are introduced in the following subsections.

\section{B. FEM controller design}

The object of this section is to design a closed-loop bending controller for the FE model so that the tip of the FE model $\delta_{e}$ converges to its desired value $\delta_{d}{ }^{3}$ We employ the optimization-based approach to compute the control inputs. The optimization problem can be defined as $\lambda=\arg \min \Gamma(\lambda)$ where $\Gamma(\lambda)$ is the objective function:

$$
\Gamma(\lambda)=\frac{1}{2}\left\|\mathbf{W}_{e} \lambda+\delta_{e}^{\text {free }}-\delta_{d}\right\|^{2}
$$

For the safe interaction with the environment, the contact force is constrained as $0 \leq \lambda_{c} \leq \lambda_{c, \text { max }}$. Besides, the constraints of the control inputs should be also considered: $\lambda_{a, \min } \leq \lambda_{a} \leq \lambda_{a, \max }$ where $\lambda_{a, \min }$ and $\lambda_{a, \max }$ are chosen based on the physical limitation.

In order to solve the constrained optimization, the numerical optimization algorithm is necessary to be employed. In order to match the standard quadratic programming (QP) formulation for the usage of software packages [24], (9) can be converted to

$$
\begin{aligned}
& \min _{\lambda_{k}} \frac{1}{2} \lambda^{T} \mathbf{Q}_{1} \lambda+\mathbf{c}_{1}^{T} \lambda \\
& \text { s.t. } \quad \mathbf{0} \leq \lambda \leq \lambda_{\text {max }} \\
& \text { and } \quad \mathbf{0} \leq \delta_{c} \perp \lambda_{c} \geq \mathbf{0}
\end{aligned}
$$

where $\lambda_{\max }=\left[\begin{array}{ll}\lambda_{a, \max } & \lambda_{c, \max }\end{array}\right]^{T}, \mathbf{Q}_{1}=\mathbf{W}_{e}^{T} \mathbf{W}_{e}$ and $\mathbf{c}_{1}=$ $\mathbf{W}_{e}^{T}\left(\delta_{e}^{\text {free }}-\delta_{d}\right)$. For the optimization, both the control inputs and the contact forces are treated as optimization variables. The linear complementarity problem (LCP) (12) is employed to capture the contact constraint.

In the following, we show a simplified method to solve the QP problem with LCP.

Finding a solution to the LCP (12) is associated with minimizing the quadratic function

$$
f\left(\lambda_{c}\right)=\lambda^{T} \mathbf{W}_{c}^{T} \lambda_{c}+\left(\delta_{c}^{\text {free }}\right)^{T} \lambda_{c}
$$

subject to the constraints $\mathbf{W}_{c} \lambda+\delta_{c}^{\text {free }} \geq 0$ and $\lambda_{c} \geq 0$.

\footnotetext{
${ }^{3}$ The variables in this subsection represent the variables for the FE model. However, we use the same variables for the real robot in Section IV.C.
}

Considering that $\lambda_{c}=\left[\begin{array}{ll}\mathbf{0}_{c \times a} & \mathbf{I}_{c \times c}\end{array}\right] \lambda$, the quadratic function (13) can be rewritten as

$$
f(\lambda)=\lambda^{T} \mathbf{Q}_{l c c} \lambda+\mathbf{c}_{l c c}^{T} \lambda
$$

where $\quad \mathbf{Q}_{l c c}=\left[\begin{array}{ll}0_{(a+c) \times a} & \mathbf{W}_{c}^{T}\end{array}\right] \quad$ and $\quad \mathbf{c}_{l c c}=$ $\left[\begin{array}{ll}0_{1 \times a} & \delta_{c}^{\text {free }}\end{array}\right]^{T}$.

Then, the QP problem with a LCP (10) can be separated into two standard QP problems:

$$
\begin{aligned}
& \mathrm{QP} 1: \min _{\lambda} \frac{1}{2} \lambda^{T} \mathbf{Q}_{1} \lambda+\mathbf{c}_{1}^{T} \lambda \\
& \text { s.t. } \quad \mathbf{0} \leq \lambda \leq \lambda_{\max }
\end{aligned}
$$

and

$$
\begin{array}{ll}
\mathrm{QP} 2: & \min _{\lambda} \frac{1}{2} \lambda^{T} \mathbf{Q}_{2} \lambda+\mathbf{c}_{2}^{T} \lambda \\
& \text { s.t. } \quad \mathbf{W}_{c} \lambda+\delta_{c}^{\text {free }} \geq 0 \\
& \text { and } \quad \lambda \geq 0
\end{array}
$$

where $\mathbf{Q}_{2}=\mathbf{Q}_{l c c}+\mathbf{Q}_{l c c}^{T}$ (Because $\lambda^{T} \mathbf{Q}_{l c c} \lambda$ is a scalar, we have $\left(\lambda^{T} \mathbf{Q}_{l c c} \lambda\right)^{T}=\lambda^{T} \mathbf{Q}_{l c c}^{T} \lambda=\lambda^{T} \mathbf{Q}_{l c c} \lambda$.) and $\mathbf{c}_{2}=\mathbf{c}_{l c c}$. The QP problem (16) corresponds to the LCP.

Combining both (10) and (16), the QP problem with the LCP (10)can be converted into a standard QP problem without the LCP:

$$
\begin{array}{ll}
\text { converted QP : } & \min _{\lambda} \frac{1}{2} \lambda^{T} \mathbf{Q} \lambda+\mathbf{c}^{T} \lambda \\
& \text { s.t. } \mathbf{0} \leq \lambda \leq \lambda_{\max } \\
& \text { and } \quad \mathbf{W}_{c} \lambda+\delta_{c}^{\text {free }} \geq \mathbf{0}
\end{array}
$$

where $\mathbf{Q}=\mathbf{Q}_{1}+\varepsilon \mathbf{Q}_{2}$ and $\mathbf{c}=\mathbf{c}_{1}+\varepsilon \mathbf{c}_{2}$ with $\varepsilon$ being the weight of the LCP.

For general problems, the parameter $\varepsilon$ should be chosen as a large value to make sure that the LCP holds during the optimization. Unlike former solvers [25], [26], the proposed strategy has easier background, faster computation and can employ standard QP solvers to solve the problem of QP with the LCP. However, we make the assumption that the defined trajectory is always reachable. In practice, with assumption of static environment, we plan it off-line and make sure that it is always reachable by our robot. For the case with a reachable trajectory, the solution of (17) is equal to the solution of (10) if $\varepsilon>0$ (The proof is presented as the appendix.).

\section{Robot controller design}

The quasi-static equations (5) and (6) can be converted as the discrete-time kinematic equations [9] ( $k$ is the timestep index):

$$
\begin{array}{r}
\delta_{e, k+1}=\delta_{e, k}+\mathbf{W}_{e a, k} \triangle \lambda_{a, k} \\
\delta_{a, k+1}=\delta_{a, k}+\mathbf{W}_{a a, k} \triangle \lambda_{a, k}
\end{array}
$$

Combining (18) and (19), we have

$$
\delta_{e, k+1}=\delta_{e, k}+\mathbf{J}_{a, k} \triangle \delta_{a, k}
$$

where $\mathbf{J}_{a, k}=\mathbf{W}_{e a, k}\left(\mathbf{W}_{a a, k}\right)^{-1}$ is the Jacobian matrix of the 


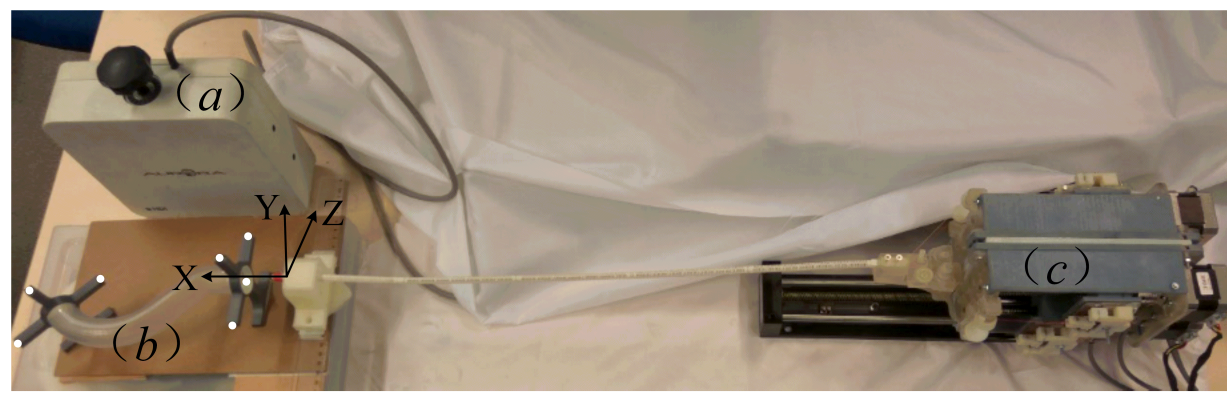

Fig. 4. Experimental setup for the motion control of a catheter robot through contacts. $(a)$ NDI electromagnetic tracking system: Aurora; $(b)$ a rigid tube for the experiment. The white points are the feature points which are used for the calibration; $(c)$ the cable-driven continuum catheter robot which has four step motors to actuate the bending and one step motor to control the insertion.

catheter robot.

As shown in Section III, it is not necessary to consider the contact information for the the controller design. Therefore, the robot controller is designed for the bending control of catheter robot based on the kinematic equation (20). It allows to control the position of catheter robot by changing the displacements of actuators.

By defining a new control vector $U_{k}=\mathbf{J}_{a, k} \triangle \boldsymbol{\delta}_{a, k}$, the kinematic model can be rewritten as $\delta_{e, k+1}=\delta_{e, k}+U_{k}$. The tracking error is defined as $e_{k}=\delta_{e, k}-\delta_{d, k}$. In the task space, the control vector $U_{k}$ can be designed as:

$$
U_{k}=-K_{P} e_{k}-K_{D}\left(e_{k}-e_{k-1}\right)
$$

where $K_{P}$ and $K_{D}$ are constant parameters for the proportional gain and differential gain, respectively.

Then, the Pseudo-inverse based control allocation is employed to obtain a unique solution:

$$
u=\mathbf{J}_{a, k}^{+} U_{k}
$$

which is the minimum 2-norm solution of $U_{k}=\mathbf{J}_{a, k} \triangle \boldsymbol{\delta}_{a, k}$ and $u=\triangle \boldsymbol{\delta}_{a, k}$ is the control input of the robot. Considering that the matrix $\mathbf{J}_{a, k}$ has full row rank (There are more actuators than effectors in our experiment), the Pseudo-inverse matrix $\mathbf{J}_{a, k}^{+}$can be computed by $\mathbf{J}_{a, k}^{+}=\mathbf{J}_{a, k}^{T}\left(\mathbf{J}_{a, k} \mathbf{J}_{a, k}^{T}\right)^{-1}$.

Substituting (21) into the (22), the control law can be written as:

$$
u=-\mathbf{J}_{a, k}^{+}\left[K_{P} e_{k}+K_{D}\left(e_{k}-e_{k-1}\right)\right]
$$

Remark 2. Considering the contacts, the kinematic equation is written as $\triangle \delta_{e}=\mathbf{J}_{a} \triangle \lambda_{a}+\mathbf{J}_{c} \triangle \lambda_{c}$ where $\mathbf{J}_{c}$ is defined as the contact Jacobian matrix and the Jacobian matrix $\mathbf{J}_{a}$ only depends on the robot shape. The kinematic equation can also be written as $\triangle \delta_{e}=\left(\mathbf{J}_{a}+\omega\right) \triangle \lambda_{a}=\mathbf{J} \triangle \lambda_{a}$ where $\omega \triangle \lambda_{a}=$ $\mathbf{J}_{c} \triangle \lambda_{c}$. In the bending space, $\mathbf{J}$ is not inverted by the contacts. Therefore, based on the assumption that we have an accurate $\mathbf{J}_{a}$ (accurate FE model of the robot shape), we can design the bending controller (20). If the FE model has a lower accuracy, the condition for a stable loop using (23) is that the error of $\mathbf{J}_{a}$ does not generate an inverted mapping of $\mathbf{J}_{a}$. Then, we can increase the robustness of the system by choosing a suitable control parameters [10].

\section{EXPERIMENT RESULTS USING DECOUPLED CONTROL}

In this section, we first introduce the experimental setup for the validation of the proposed decoupled control strategy. Then, we show the experiment results of trajectory tracking and positioning for a cable-driven continuum catheter robot. Besides, we validate the collision detection and contact forces estimation by experiment using a soft rod. Finally, we test respectively the decoupled control with Jacobian matrix rotation and the trajectory tracking through a soft tube by simulation.

\section{A. Experimental setup}

The experimental setup for the position control of a cabledriven catheter robot through contacts is shown in Fig. 4. Three components are needed for the implementation of the experiments: a position tracking system $(a)$, an obstacle to generate the contacts between catheter robot and its environment $(b)$, and a continuum catheter robot $(c)$.

The commercial electromagnetic tracking system is employed (Aurora by NDI company) with the sampling frequency $20 \mathrm{~Hz}$ and the sub-millimetric accuracy. A rigid tube (diameter 28mm) is fixed with respect to the base of the catheter robot. For the calibration of coordinate systems between the Aurorat and the catheter robot, we choose two corresponding sets (seven points for each set) of keypoints defined for the rigid tube. The positions of the keypoints are measured respectively from the Aurora system and the FE model. Then, the transformation between two sets of points can be solved robustly using the Singular Value Decomposition (SVD) method.

The movement of catheter robot consists of bending and insertion. The bending is controlled by four cables using four step motors and the insertion is actuated using one step motor. The robot is a modified version in [18]. The structure of the catheter robot (diameter $7 \mathrm{~mm}$ ) is made of polymer material which is MR-compatible and is manufactured using the rapid prototyping technique. Both the robot and the rigid tube are simulated in SOFA framework (a real-time FEM simulation software). The frequency of the simulation can reach to $50 \mathrm{~Hz}$ in our experiment (100 mesh nodes). The detail strategy for FEM modeling can be seen in [23]. 


\section{B. Trajectory Tracking Results}

The central line of the tube is defined as the desired trajectory for the robot tip. The tracking performances are illustrated in Fig. 5 where both the desired trajectory and the catheter tip trajectory are projected to the $\mathrm{XZ}$ plane and $\mathrm{XY}$ plane. In our experiments, the desired trajectory consists of twice bending along the insertion direction. Three different speeds of insertion (For each case, the insertion speed is uniform.) are tested in the experiments. We choose the same control parameters for all cases, $K_{P}=0.2$ and $K_{D}=0.002$.
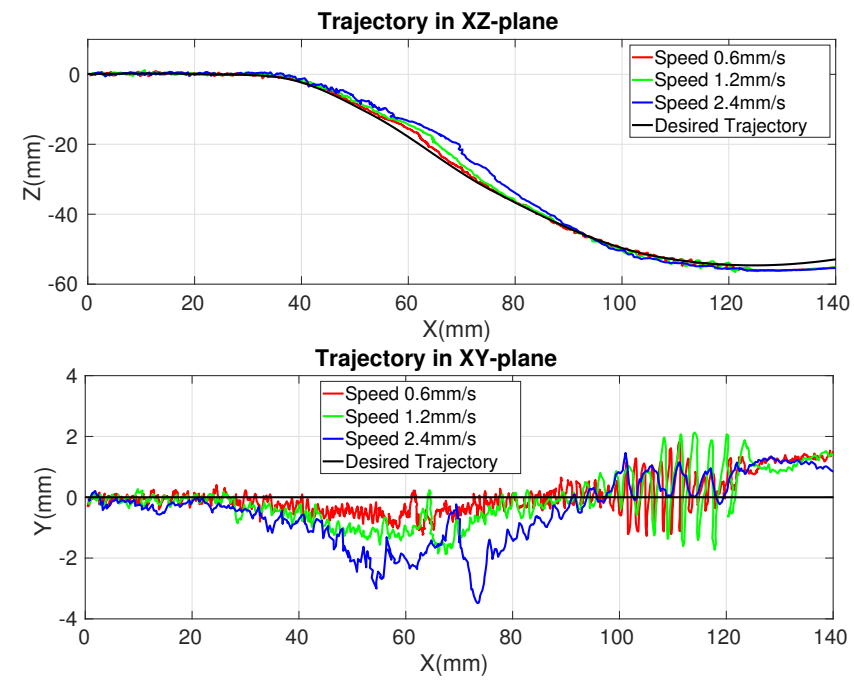

Fig. 5. Trajectories of catheter tip in $\mathrm{XZ}$ plane and in $\mathrm{XY}$ plane with different speeds of insertion $(0.6 \mathrm{~mm} / \mathrm{s}, 1.2 \mathrm{~mm} / \mathrm{s}$ and $2.4 \mathrm{~mm} / \mathrm{s})$.

The tracking errors are defined as the distance between the catheter tip and its desired position. We compute the tracking error based on the positions on the bending plane. As shown in Fig. 6, the average tracking error is around $0.3 \mathrm{~mm}$ when the desired trajectory is a straight line. However, the higher insertion speed deteriorates the tracking performances when there is a bending along the desired trajectory. The largest error increases to be $2 \mathrm{~mm}, 3.2 \mathrm{~mm}$ and $4.8 \mathrm{~mm}$ respectively for the insertion speeds $0.6 \mathrm{~mm} / \mathrm{s}, 1.2 \mathrm{~mm} / \mathrm{s}$ and $2.4 \mathrm{~mm} / \mathrm{s}$. The error increases with the increment of the insertion speed because the lower insertion speed allows longer time for the convergence of the tip position. For the application, the insertion is controlled manually and its speed should be lower when the catheter robot starts to bend.

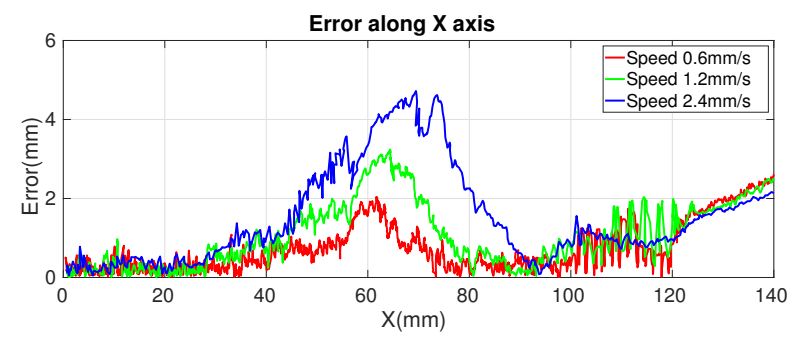

Fig. 6. Trajectory errors of catheter tip with different speeds of insertion.

\section{Positioning Results}

The positioning results are shown in Fig. 7 which includes two cases with different catheter length $60 \mathrm{~mm}$ and $166 \mathrm{~mm}$ ). The tracking error converges faster when the length of catheter is shorter. For both cases, the positioning errors converge to a small bound (around $0.2 \mathrm{~mm}$ ). Usually a longer length of catheter robot generates a larger convergence bound if there is no interaction with the environment. However, in our experiments, the contacts limit the deflection and therefore reduce the convergence bound.

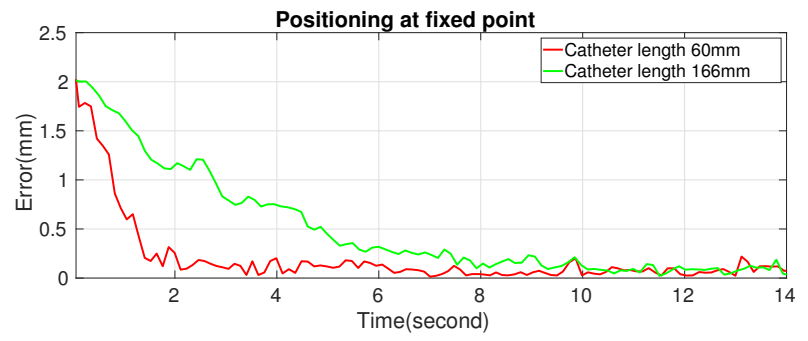

Fig. 7. Convergence of positioning error with different catheter length $(60 \mathrm{~mm}$ and $166 \mathrm{~mm})$.

\section{Validation of contact forces estimation}

As shown in Fig. 8, three experiments are designed to validate respectively the collision detection, the accuracy of FE model and the contact force computation. The collision is detected based on the relative positions between the contact and the soft rod. The contact positions are very close for both the FE model and the real system (see $(a)$ and $(b)$ in Fig. 8). The FE model is tested using a 3D tube (see $(c)$ and $(d)$ in Fig. 8). Ten static positions are measured with different insertions and compared between the real system and the FE model. The positions are drawn in Fig. 9 and the average position error is $3.35 \mathrm{~mm}$ in our experiment. The accuracy of the FE model ensures the success to capture the relation between the insertion and the tip position.

The computation of contact force is validated using the setup $(e)$ and $(f)$ in Fig. 8. The length of the soft rod is $210 \mathrm{~mm}$ and its Young's Modulus is $1.4 \mathrm{GPa}$. Nine groups of forces are measured and computed with different target tip positions and different contact points. The measured forces using a force sensor and the computed forces using the FE method are listed in Tab. I. The accuracy of contact force computation can reach to $97.1 \%$.

TABLE I

VALIDATION OF CONTACT FORCE COMPUTATION (T: TARGET, C: Contact, M: Measured Force, E: Estimated Force). The Unit of THE FORCES IS $m N$.

\begin{tabular}{|c|c|c|c|c|c|c|c|c|c|}
\hline T & \multicolumn{3}{|c|}{ B1 } & \multicolumn{3}{c|}{ B2 } & \multicolumn{3}{c|}{ B3 } \\
\hline C & A1 & A2 & A3 & A1 & A2 & A3 & A1 & A2 & A3 \\
\hline M & 110 & 198 & 372 & 216 & 357 & 682 & 336 & 537 & 998 \\
\hline E & 119 & 193 & 380 & 214 & 345 & 679 & 324 & 527 & 1030 \\
\hline
\end{tabular}

The contact forces computed based on the FE model can be regarded as the approximation of the contact forces for the catheter robot. However, our contribution here is based 


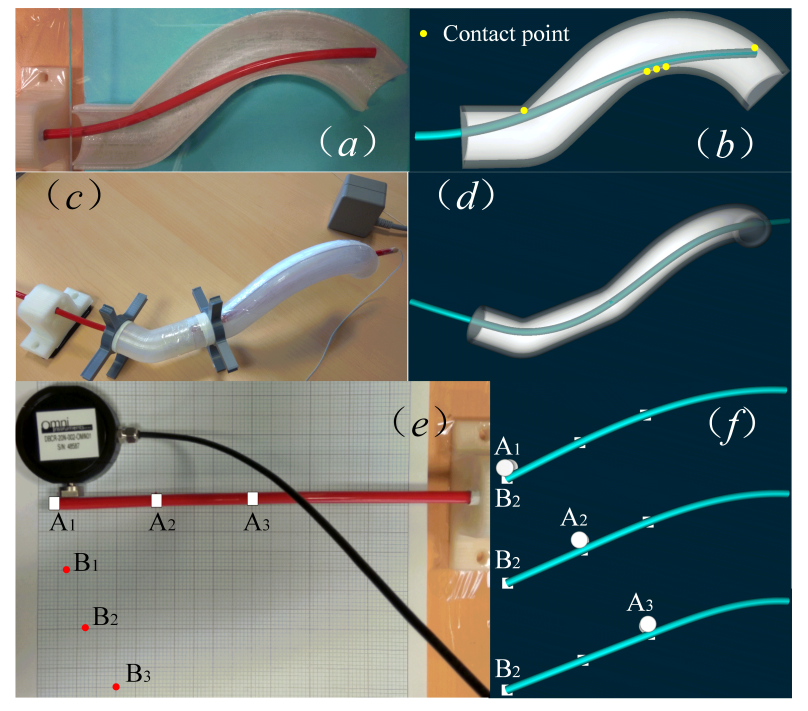

Fig. 8. Experiment setup for the validation of the contact detection $((a)$ and $(b))$, the accuracy of simulation $((c)$ and $(d))$ and the contact force computation $((e)$ and $(f))$. In $(e)$ and $(f), \mathrm{A} 1-\mathrm{A} 3$ and $\mathrm{B} 1-\mathrm{B} 3$ are respectively the contact locations and the desired positions of the tip.
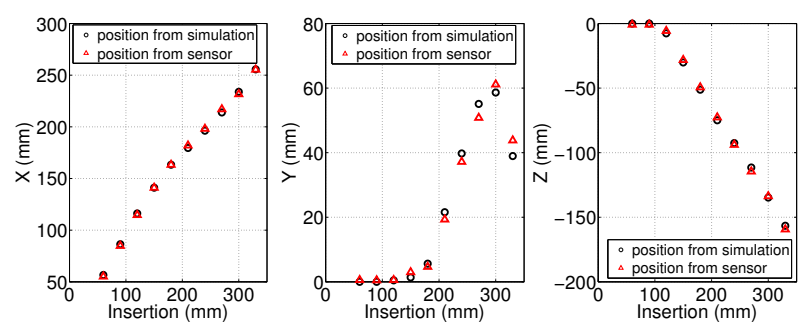

Fig. 9. Comparison of tip positions for the experiment in Fig. 8 ((c) and $(d))$.

on the assumption that the boundary conditions are fully and perfectly known. To simplify the experiment, we validate our method in the situation of single contact.

\section{E. Simulation-based validation}

The simulation-based validation allow to focus on the control algorithms themselves and can get rid of the sensor noise, imprecise modeling of robot and contacts. In this subsection, we first validate the decoupled control strategy to deal with the problem of the Jacobian matrix rotation. Then, we show the simulation result when the catheter robot interacts with a soft tube.

1) Control strategy to deal with the Rotation of Jacobian Matrix: As shown in Fig. 10, the catheter robot can be controlled from the initial configuration $(a)$ to the finial configuration $(b)$ with the decoupled control strategy. If the insertion and bending are coupled between each other, the Jacobian matrix is inverted during the movement. However, the decoupled control strategy can successfully avoid the problem of the Jacobian matrix rotation when the robot interacts with the contacts.

2) decoupled control with soft tube: The trajectory tracking can also be achieved when the catheter robot interacts with soft contacts. The decoupled control strategy is em-

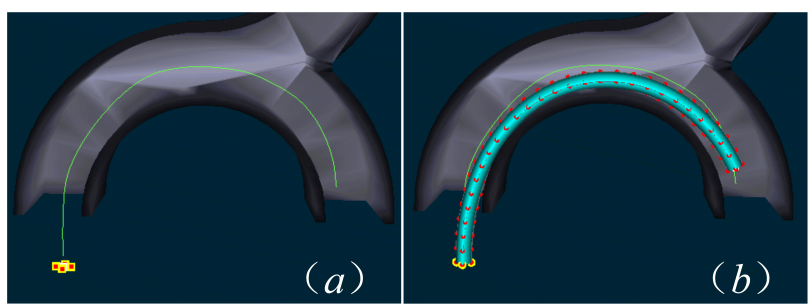

Fig. 10. Simulation-based control validation for catheter robot through contacts. $(a)$ and $(b)$ are respectively the initial configuration and the finial configuration of the catheter robot.

ployed and validated using simulation which is shown in Fig. 11. To estimate the Jacobian matrix and contact forces, both catheter robot and soft tube are modeled using FE method and are simulated in real-time.

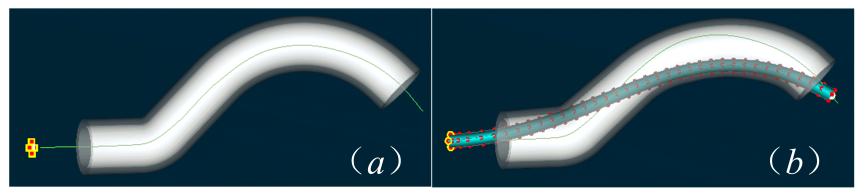

Fig. 11. Simulation-based validation of decoupled control of catheter robot through a soft tube. (a) The initial state of catheter robot and the soft tube; (b) The simulated deformation of the soft tube and the robot.

\section{CONCLUSION}

This work contributes to the position control of catheter robot in the constrained space. With the aim to provide safe insertion for the catheter robot, in this paper, a decoupled controller is designed and allows to control the bending and the insertion independently. The proposed strategy can achieve a stable position control and can avoid the problem of Jacobian matrix rotation. Besides, the decoupled control allows the surgeon to remain in direct control of the catheter insertion. As a benefit of the proposed method, the contact forces along the entire catheter robot can be computed without using force sensors. So, during the insertion, it is possible to provide to the surgeon a feedback on the force intensity applied on the entire catheter. A simplified method to compute quadratic programming problem with a linear complementarity problem is proposed in our work. The proposed strategy is general and the application is not limited for the cable-driven catheter robot. We perform some experiments and some simulations to test our methods. In the following, we address the main limitations of this work, possible solutions and future works.

To estimate the contact forces, we need the assumption that we have an accurate model of the contact and the catheter robot. However, it is very difficult to always fulfill this assumption in complex situation of real applications. When the contact objects are not modeled well, the registration method for deformation structures [27] could be employed to improve the accuracy of FE model.

Besides, to make the tracking error as small as possible, the insertion speed should be limited in a small value. When the catheter robot starts to bend, the tracking error increases and needs relatively longer time to converge. Instead of limiting 
the insertion speed in a quite small range, we could use adaptive control parameters for the control law to increase the convergence speed.

In the future, we would also like to explore the hybrid force and position control for the catheter robot and improve the control system by registration of both contacts and catheter robot with their FE models. Finally, the proposed techniques should be validated in vivo.

\section{APPENDIX}

It is assumed that $F_{1}, F_{2}, F_{3}$, and $F_{4}$ are the minimum value of objective functions for (10), (15), (16) and (17), respectively. $\lambda_{1}, \lambda_{2}, \lambda_{3}$ and $\lambda_{4}$ are respectively the solution of QP problems (10), (15), (16) and (17), respectively. Then, a corollary about the proposed approach is provided as follows:

Corollary: If the desired position is reachable (the solution of (9) is zero), the solution of (17) is equal to the solution of (10).

proof: The proof consistsof two steps:

Step 1: $\lambda_{1}$ is the solution of the QP problem (17).

For the QP problem (10) with the solution $\lambda_{1}$, we have $F_{1}=-\frac{1}{2}\left\|\delta_{e}^{\text {free }}-\delta_{d}\right\|^{2}$ and the LCP (12) holds so that, for QP problem (16) with $\lambda_{3}=\lambda_{1}$, we have $F_{3}=0$. Then, for the QP problem (15) with $\lambda_{2}=\lambda_{1}$, we have $F_{2}=-\frac{1}{2}\left\|\delta_{e}^{\text {free }}-\delta_{d}\right\|^{2}$. Therefore, the objective functions (17) has the same minimum value $F_{4}=-\frac{1}{2}\left\|\delta_{e}^{\text {free }}-\delta_{d}\right\|^{2}$ and $\lambda_{4}=\lambda_{1}$ is the solution of the QP problem (17).

Step 2: $\lambda_{4}$ is the solution of the QP problem (10).

Because the objective function (17) is the sum of (15) and (16) and have a more strict constraint, we have the conclusion that $F_{4} \geq F_{2}+F_{3} \geq-\frac{1}{2}\left\|\delta_{e}^{\text {free }}-\delta_{d}\right\|^{2}$. If the desired position is reachable, there is solution $\lambda_{4}$ for (17) so that its objective function has the minimum value: $F_{4}=-\frac{1}{2}\left\|\delta_{e}^{\text {free }}-\delta_{d}\right\|^{2}$. Then, we have $F_{2}=-\frac{1}{2}\left\|\delta_{e}^{\text {free }}-\delta_{d}\right\|^{2}$ with $\lambda_{2}=\lambda_{4}$, and $F_{3}=0$ with $\lambda_{3}=\lambda_{4}$. Therefore, $\lambda_{4}$ is a solution to make (10) reach the minimum value and make (12) hold. Then, we can conclude that $\lambda_{1}=\lambda_{4}$ is the solution of the QP problem (10).

\section{REFERENCES}

[1] M. Khoshnam and R. V. Patel, "A pseudo-rigid-body 3r model for a steerable ablation catheter," in Robotics and Automation (ICRA), 2013 IEEE International Conference on, pp. 4427-4432, IEEE, 2013.

[2] T. Greigarn and M. C. Çavuşoğlu, "Task-space motion planning of mriactuated catheters for catheter ablation of atrial fibrillation," in Intelligent Robots and Systems (IROS 2014), 2014 IEEE/RSJ International Conference on, pp. 3476-3482, IEEE, 2014.

[3] W. Tang, T. R. Wan, D. A. Gould, T. How, and N. W. John, "A stable and real-time nonlinear elastic approach to simulating guidewire and catheter insertions based on cosserat rod," IEEE Transactions on Biomedical Engineering, vol. 59, no. 8, pp. 2211-2218, 2012.

[4] L. B. Kratchman, T. L. Bruns, J. J. Abbott, and R. J. Webster, "Guiding elastic rods with a robot-manipulated magnet for medical applications," IEEE Transactions on Robotics, vol. 33, no. 1, pp. 227-233, 2017.

[5] Y. Ganji, F. Janabi-Sharifi, and A. N. Cheema, "Robot-assisted catheter manipulation for intracardiac navigation," International journal of computer assisted radiology and surgery, vol. 4, no. 4, pp. 307-315, 2009.

[6] C. Duriez, S. Cotin, J. Lenoir, and P. Neumann, "New approaches to catheter navigation for interventional radiology simulation," Computer aided surgery, vol. 11, no. 6, pp. 300-308, 2006.
[7] Y. Bailly, Y. Amirat, and G. Fried, "Modeling and control of a continuum style microrobot for endovascular surgery," IEEE Transactions on Robotics, vol. 27, no. 5, pp. 1024-1030, 2011.

[8] P. M. Loschak, L. J. Brattain, and R. D. Howe, "Algorithms for automatically pointing ultrasound imaging catheters," IEEE Transactions on Robotics, vol. 33, no. 1, pp. 81-91, 2017.

[9] Z. Zhang, J. Dequidt, A. Kruszewski, F. Largilliere, and C. Duriez, "Kinematic modeling and observer based control of soft robot using real-time finite element method," in Intelligent Robots and Systems (IROS), 2016 IEEE/RSJ International Conference on, pp. 5509-5514, IEEE, 2016

[10] Z. Zhang, T. M. Bieze, J. Dequidt, A. Kruszewski, and C. Duriez, "Visual servoing control of soft robots based on finite element model," in IROS 2017-IEEE/RSJ International Conference on Intelligent Robots and Systems, 2017.

[11] M. C. Yip and D. B. Camarillo, "Model-less feedback control of continuum manipulators in constrained environments," IEEE Transactions on Robotics, vol. 30, no. 4, pp. 880-889, 2014.

[12] M. C. Yip, J. A. Sganga, and D. B. Camarillo, "Autonomous control of continuum robot manipulators for complex cardiac ablation tasks," Journal of Medical Robotics Research, vol. 2, no. 01, p. 1750002, 2017.

[13] J. Back, L. Lindenroth, K. Rhode, and H. Liu, "Model-free position control for cardiac ablation catheter steering using electromagnetic position tracking and tension feedback," Frontiers in Robotics and AI, vol. 4, p. 17, 2017.

[14] P. Polygerinos, A. Ataollahi, T. Schaeffter, R. Razavi, L. D. Seneviratne, and $\mathrm{K}$. Althoefer, "Mri-compatible intensity-modulated force sensor for cardiac catheterization procedures," IEEE Transactions on biomedical engineering, vol. 58, no. 3, pp. 721-726, 2011.

[15] T. N. Do, T. E. T. Seah, and S. J. Phee, "Design and control of a mechatronic tracheostomy tube for automated tracheal suctioning," IEEE Transactions on Biomedical Engineering, vol. 63, no. 6, pp. 12291238, 2016.

[16] W. Wei and N. Simaan, "Modeling, force sensing, and control of flexible cannulas for microstent delivery," Journal of Dynamic Systems, Measurement, and Control, vol. 134, no. 4, p. 041004, 2012.

[17] M. Khoshnam, A. C. Skanes, and R. V. Patel, "Modeling and estimation of tip contact force for steerable ablation catheters," IEEE Transactions on Biomedical Engineering, vol. 62, no. 5, pp. 1404-1415, 2015.

[18] J. Back, L. Lindenroth, K. Rhode, and H. Liu, "Three dimensional force estimation for steerable catheters through bi-point tracking," Sensors and Actuators A: Physical, vol. 279, pp. 404-415, 2018.

[19] C. Duriez, C. Andriot, and A. Kheddar, "Signorini's contact model for deformable objects in haptic simulations," in Intelligent Robots and Systems, 2004.(IROS 2004). Proceedings. 2004 IEEE/RSJ International Conference on, vol. 4, pp. 3232-3237, IEEE, 2004.

[20] H. Courtecuisse, J. Allard, P. Kerfriden, S. P. Bordas, S. Cotin, and C. Duriez, "Real-time simulation of contact and cutting of heterogeneous soft-tissues," Medical image analysis, vol. 18, no. 2, pp. 394410, 2014.

[21] M. Posa, C. Cantu, and R. Tedrake, "A direct method for trajectory optimization of rigid bodies through contact," The International Journal of Robotics Research, vol. 33, no. 1, pp. 69-81, 2014.

[22] E. Coevoet, A. Escande, and C. Duriez, "Optimization-based inverse model of soft robots with contact handling," IEEE Robotics and Automation Letters, vol. 2, no. 3, pp. 1413-1419, 2017.

[23] E. Coevoet, T. Morales-Bieze, F. Largilliere, Z. Zhang, M. Thieffry, M. Sanz-Lopez, B. Carrez, D. Marchal, O. Goury, J. Dequidt, et al., "Software toolkit for modeling, simulation, and control of soft robots," Advanced Robotics, vol. 31, no. 22, pp. 1208-1224, 2017.

[24] H. J. Ferreau, C. Kirches, A. Potschka, H. G. Bock, and M. Diehl, "qpoases: A parametric active-set algorithm for quadratic programming," Mathematical Programming Computation, vol. 6, no. 4, pp. 327-363, 2014.

[25] L. Chen, D. Goldfarb, et al., "An active-set method for mathematical programs with linear complementarity constraints," Manuscript, Department of Industrial Engineering and Operations Research, Columbia University, 2007.

[26] L. Bai, J. E. Mitchell, and J.-S. Pang, "On convex quadratic programs with linear complementarity constraints," Computational Optimization and Applications, vol. 54, no. 3, pp. 517-554, 2013.

[27] I. Peterlík, H. Courtecuisse, R. Rohling, P. Abolmaesumi, C. Nguan, S. Cotin, and S. Salcudean, "Fast elastic registration of soft tissues under large deformations," Medical Image Analysis, vol. 45, pp. 2440, 2018. 